\title{
BioSpotlight
}

\section{Archimedes' Ants}

Physiological, evolutionary, and ecological studies of the scaling of body parts depend on accurate volume data, yet this information is not widely available for small or irregularly shaped biological structures with complex geometric shapes. Such data can be difficult to collect as studies are often conducted on samples in remote locations, precluding the use of sophisticated or delicate instruments. While volume data may be acquired using a microbalance to measure mass or by reconstructing estimated volumes based on histological sections, these methods are time-consuming, difficult, or expensive. Histological methods can also be less reliable due to sample shrinking and other artifacts caused by the dehydration and processing steps needed for paraffin embedding. To address the need for a portable, rapid, and inexpensive means of collecting volume data, J.K. Douglass and W.T. Wcislo report the development of an enhanced microvolumeter for measuring volumes of small biological samples such as zooplankton, embryos, small animals, and organs. Their method is based on Archimedes' principle, which states that adding an arbitrarily shaped object to a fluid-filled container displaces an equivalent fluid volume.
The microvolumeter consists of a fluid-filled receptacle connected to a syringe that can be used to adjust the fluid levels to a fixed position where the sample volume will equal the difference between the syringe plunger's position before and after addition of the sample. Since small samples result in small changes in fluid levels, the authors used reflected light aimed at the top edge of the receptacle and optimized ratios of sample volume to container diameter to enhance accuracy and precision. They also took a series of measurements over time to account for any possible evaporation. To demonstrate its utility, the volumes of ant brains were measured with the new device and compared to measurements acquired using confocal microscopy. The results show that volume measurements between $0.066 \mu \mathrm{L}$ and $1 \mathrm{~mL}$ could be accurately obtained using the microvolumeter, thus allowing accurate measurement of samples in less time and without the need for the fixation and clearing of samples required for confocal microscopy.

See "An inexpensive and portable microvolumeter for rapid evaluation of biological samples" on page 566.

\section{A Little 1-to-1 QD Time}

Direct labeling of receptor proteins can be accomplished through covalent attachment of fluorophores to reactive amino acid side chains or by conjugation to fusion protein tags that react with modified fluorophores. While such labeling is routinely carried out with organic fluorophores, it has not previously been done using quantum dots (QDs), which provide superior fluorescent signaling due to their greater brightness and resistance to photobleaching. In this issue, G. Harms and colleagues at the University of Würzburg (Würzburg, Germany) describe their new method-based on the acyl carrier protein (ACP) system - for the direct covalent labeling of receptors with QDs in a 1:1 ratio using a short linker. The ACP extracellular protein fusion tag is wellsuited for this application as it is smaller (77 amino acids) than comparable tags such as CLIC and SNAP, reacts specifically and efficiently with co-enzyme A (CoA) bound to organic fluorophores in a reaction catalyzed by phosphopantetheinyl transferase (PPTase), and doesn't interfere with the biological activity of the membrane protein it is fused with. The key innovation of this new technique is the covalent labeling of $\mathrm{CoA}$ with commercially available polymer-coated $\mathrm{CdSe} / \mathrm{ZnS}$ QDs using thiol-reactive chemistry to achieve a maximal QD:CoA

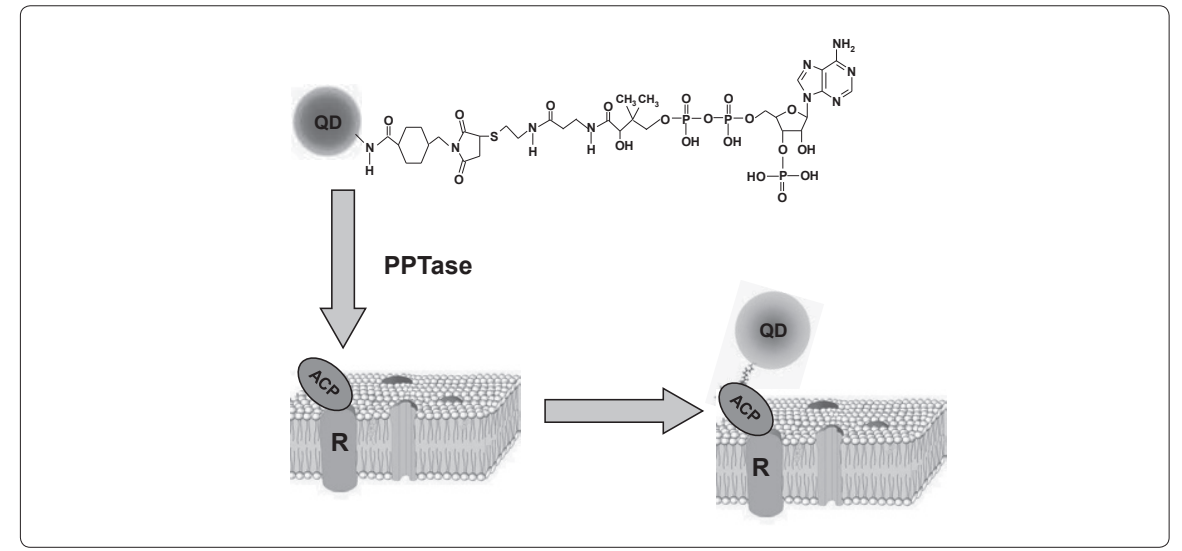

Schematic diagram illustrating the covalent attachment catalyzed by PPTase of the QD-functionalized CoA to the ACP fusion protein tag on the extracellular surface of a receptor.

ratio of $1: 1$. To validate their method, the authors examined truncation mutants of the parathyroid hormone receptor and the bone morphogenetic protein receptor type II that were fused to ACP and transfected into HEK293 cells. Efficient and specific QD labeling of receptor-ACP fusion proteins by CoA-QD was observed only in the presence of PPTase. Using total internal reflection (TIRF) and widefield microscopy, it was possible to clearly track the lateral diffusion and internalization behavior of labeled receptors with single molecule resolution. In the future, the authors plan to improve their method through the use of a 12 amino acid-long CoA binding domain and the application of smaller, more biologically inert QDs.

See "Covalent quantum dot receptor linkage via the acyl carrier protein for single-molecule tracking, internalization, and trafficking studies" on page $\mathbf{5 7 4 .}$

Selected and written by Patrick C.H. Lo,

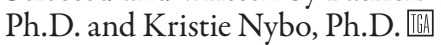

BioTechniques 49:541 (August 2010)

doi $10.2144 / 000113471$ 